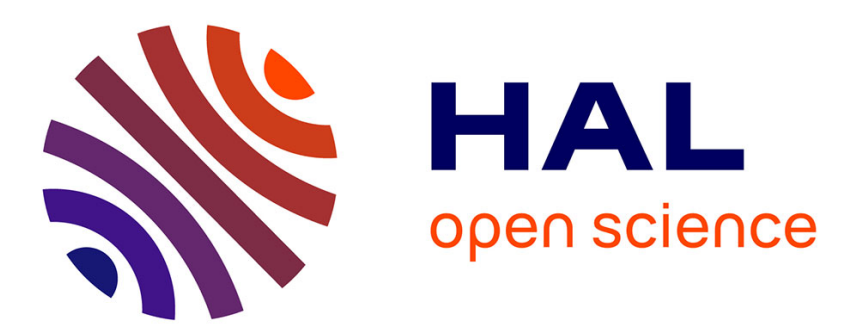

\title{
Performance Evaluation of an Active Signaling based Time-Slot Scheduling Scheme for connected vehicles
}

Fouzi Boukhalfa, Mohamed Hadded, Paul Mühlethaler, Oyunchimeg Shagdar

\section{To cite this version:}

Fouzi Boukhalfa, Mohamed Hadded, Paul Mühlethaler, Oyunchimeg Shagdar. Performance Evaluation of an Active Signaling based Time-Slot Scheduling Scheme for connected vehicles. Annals of Telecommunications - annales des télécommunications, 2020, 10.1007/s12243-020-00818-8 . hal$02981063 \mathrm{v} 2$

\section{HAL Id: hal-02981063 \\ https://hal.science/hal-02981063v2}

Submitted on 29 Oct 2020

HAL is a multi-disciplinary open access archive for the deposit and dissemination of scientific research documents, whether they are published or not. The documents may come from teaching and research institutions in France or abroad, or from public or private research centers.
L'archive ouverte pluridisciplinaire HAL, est destinée au dépôt et à la diffusion de documents scientifiques de niveau recherche, publiés ou non, émanant des établissements d'enseignement et de recherche français ou étrangers, des laboratoires publics ou privés. 


\title{
Performance Evaluation of an Active Signaling based Time-Slot Scheduling Scheme for connected vehicles
}

\author{
Fouzi Boukhalfa*‡, Mohamed Hadded*, Paul Muhlethaler ${ }^{\dagger}$ and Oyunchimeg Shagdar* \\ *Institute VEDECOM, 23 bis allée des Marronniers, 78000 Versailles, France \\ ${ }^{\dagger}$ EVA Team, Inria Paris, 2 Rue Simone Iff, 75012 Paris, France \\ ${ }^{\ddagger}$ Correspondence to fouzi.boukhalfa@vedecom.fr
}

\begin{abstract}
Latency is a very important metric to be taken into account in the design of the Connected and Automated Driving (CAD) technology. Today, connected vehicles have a dedicated technology named vehicle-to-everything (V2X). Many current research efforts and standardization activities aim to make the next generation of $\mathrm{V} 2 \mathrm{X}$ technology able to offer new usages and services, with the main focus being on supporting road safety applications: hazard, obstacle and collision avoidance, etc. To satisfy the real-time constraints of safety applications, Time Division Multiple Access (TDMA) is a widely-used technique to control and share the channel. TDMA-based Medium Access Control (MAC) protocols have resolved many issues in VANETs and shown good performances in the literature by ensuring a bounded access delay to send an emergency message. However, latency can be further improved by eradicating some imperfections in the functionality of these protocols, such as the access collision problem which can occur when two or more vehicles in the same two-hop neighborhood set try to access the same slot at the same time. That is why we recently proposed an Active Signaling-based TDMA MAC protocol, called ASDTMAC, which operates above the existing DTMAC protocol. In this paper, we analytically study the performance of ASDMTAC when we have a homogeneous arrival on each time slot with an error in the signaling process.

keywords - Connected vehicles, VANETs, MAC, TDMA, Active signaling, Low latency, Next-generation V2X, Analytical modeling.
\end{abstract}

\section{Context and motivation}

Vehicular mobility is increasingly facing a number of challenges. The rapid increase in traffic density, the number of road accidents (according to the world Health Organization (WHO), 1.35 million people die on the roads each year) and the lack of transportation services to make traveling more comfortable, have motivated both the research and industrial communities to invest in Vehicular Ad hoc NETworks (VANETs) [1]. VANETs are especially being developed to meet the challenges mentioned above and to have the ability to support applications for road safety, traffic management and entertainment [4]. Different types of communications can be established in VANETs, namely: Vehicle-to-Infrastructure and vice versa (V2I2V),
Vehicle-to-Vehicle (V2V) and Infrastructure to Infrastructure (I2I)[4].

The coming of autonomous driving raises new challenges in vehicular networks. In fact, some studies have shown that a large number of accidents could be avoided if the drivers were warned at least $0.5 \mathrm{~s}$ beforehand [2], [3]. This is why real time communication technologies are becoming a key factor of autonomous driving to increase the safety of drivers and passengers.

To guarantee an immediate reaction, such vehicles need to collect information from their environment through their sensors: the position and velocity of other vehicles, the presence of obstacles, etc. But at the same time, this information needs to be completed by redundant information coming from V2X communication [5]. Thus, a vehicle will be able to react safely to events that may occur suddenly. These applications require better performances than those achieved on existing networks; in particular, they require very low end-to-end latency, which can be less than $1 \mathrm{~ms}$, and a high transmission rate for huge data exchange. In addition, some use cases require ultra-reliability of $99.99 \%$ [24].

In wireless communication, the radio channel is the most precious resource. The Medium Access Control (MAC) layer has the role of sharing this expensive resource by providing efficient and reliable access to the channel for each node present in the network. In VANETs, the continuous and fast changes of network topology due to high node mobility, make the design of the MAC more difficult to handle [6]. To meet these requirements, the design of a new MAC layer is essential. Recently, several MAC protocols for VANETs have been proposed in the literature [7] [8] [9] [10], each of them treating a particular problem in a specific mobility scenario. As in traditional ad hoc networks, protocols are classified according to the control scheme used to access the channel [11], namely: contention-based or contentionfree. In contention-based, the carrier sensing scheme [12] is used to coordinate the access to the channel. Thus, the risk of collision is still possible in this category, as several vehicles may sense that the channel is free at the same time and then attempt to transmit their data simultaneously.

The current IEEE 802.11p standard [13] developed for vehicular networks is a contention-based MAC using a priority-based access scheme that employs both Enhanced Distributed Channel Access (EDCA) and Carrier Sense Multiple Access with Collision Avoidance (CSMA/CA) 
mechanisms [14]. Furthermore, the channel access time is uniformly divided into different synchronization intervals of $100 \mathrm{~ms}$ in length [15], and each of them includes two intervals: a Control Channel Interval (CCHI) and a Service Channel Interval (SCHI), each of $50 \mathrm{~ms}$ in length. Therefore, the lack of a dynamic interval in this configuration may penalize performance in terms of throughput and delay when the traffic density is high, especially for the $\mathrm{CCH}$ interval when it is not enough to transmit all the safety messages [16]. This feature makes this standard less suitable for real-time applications, while in the second category (i.e, contention-free), only one vehicle in a two-hop neighborhood set is authorized to access the channel at a given time, which reduces the risk of collision. Hence, this category of protocols is more suitable to manage real-time applications in VANETs as it can provide a bounded access delay and reliable communication for safety applications. Over the last few years, contention-free MAC protocols have remained one of the emerging areas of research and represent the majority of MAC protocols proposed in this field.

This contention-free category includes several techniques: Frequency Division Multiple Access (FDMA), Code Division Multiple Access (CDMA), and Time Division Multiple Access (TDMA). To satisfy the real-time constraints of safety applications, Time Division Multiple Access (TDMA) is a very appreciated technique to coordinate access to the channel. In TDMA, the vehicles share the same channel frequency but at different time slots that are managed in a widely-used or centralized way by updating the scheduling table. This kind of protocol gives a bounded access delay with a lower collision rate [9]. However, these schemes can suffer from access ${ }^{1}$ and merging ${ }^{2}$ collisions. Consequently, all the research activities in this field tend to focus on these problems, which can occur frequently in vehicular networks. For instance, the Medium Access Control (ADHOC MAC) protocol [17] has been proposed specially to overcome the hidden-exposed terminal problem. ADHOC MAC is a distributed contention-free scheme based on RALOHA (Reliable R-ALOHA [18]) in which each vehicle can access the channel at least once in each frame by randomly selecting a time slot as its Basic CHannel (BCH). Another wellknown contention-free MAC protocol called VeMAC [7] has been proposed to solve the problem of merging collisions, VeMAC has the particularity of assigning disjoint sets of time slots to vehicles moving in opposite directions and to Road Side Units (RSUs). Although VEMAC supports multihop broadcast services on the control channel, it suffers from the access collision problem as its scheduling is fully distributed. Recently, several solutions such as in [19], [20], [21], and [22] have attempted to improve the performance of the VEMAC protocol by dynamically adjusting the size of the slot sets according to vehicle density in each direction.

1. An access collision problem occurs when two or more vehicles within the same two-hop neighborhood set attempt to access the same available time slot, a problem which is likely to happen when a distributed scheme is used [11].

2. A merging collision problem occurs when two vehicles in different two-hop sets accessing the same time slot become members of the same two-hop set due to changes in their position
In order to satisfy the requirements of the future technology in terms of latency, we recently proposed an Active Signaling-based TDMA MAC protocol, called AS-DTMAC [9], which operates above the existing DTMAC (a fully distributed-based protocol [8]) that shows the advantages of TDMA in the sense that when a slot is acquired by a vehicle this slot can be re-used synchronously and the transmissions do not suffer from the hidden-node problem as in IEEE 802.11p. By adding the active signaling mechanism, this new protocol reduces the rate of access collisions. Moreover, simulation results show that AS-TDMAC can provide a low latency for urgent packets. Recently, we showed in another study [23], the high performances of AS-DTMAC with an analytical model based on the generating function and we validated these results using simulations. In this paper we study the entire AS-DTMAC scheme in more complex conditions than in [23]. In particular, the errors that can occur during the signaling process are fully studied. We assume that we can detect 'ghost' signaling bursts and conversely that sometimes we cannot detect real bursts. The analytical model, which takes into account these two effects, is more complex than the initial model without error; moreover, the explanation of the whole model has been largely improved. The simulation results and the analytical model show the robustness of the protocol. Moreover, we extend the simulation to show how the selection process can operate at the physical layer.

The paper is organized as follows. Section 2 recalls the DTMAC protocol and the enhanced version, AS-DTMAC with their main features. In Section 3, we introduce our analytical model and 3 case studies: homogeneous arrival, homogenous arrival with an error in the signaling process, bursts of traffic for urgent packets. Section 4 concludes this paper and discusses future work.

\section{Active signaling-based DTMAC (AS- DTMAC)}

AS-DTMAC [9] is a fully distributed TDMA, which is mainly based on the DTMAC protocol [8]. Thanks to GPS technology, each vehicle is able to have information about its position and the exact time. This information is useful for the functionality of DTMAC. The main strategy of DTMAC is to split the road into different zones $\left(x_{i}, i=1, \ldots, N\right)$ according to the communication range of the vehicles, denoted R. In this way, we can impose a new concept of slot reuse, which consists in reusing the slot spatially. As described in [8], the vehicles in zone $x_{1}$ can use the same set of slots as the vehicles in $x_{4}$. The only condition to this spatial reuse is that the distance between simultaneously transmitting vehicles must be greater than $2 \times R$. Furthermore, the slot scheduling table is updated each time a packet is sent by a vehicle. The packet sent contains a special field, named Frame Information (FI), which specifies the status of the slots. Thus, it will be easy for all vehicles to select the available slots in the next frame. DTMAC acts like a slotted Aloha protocol on the 'non-busy' slots of the frame. This protocol can be applied to highway scenarios and supports 
different parameters (vehicles moving in opposite directions, varying speeds, varying densities).

The new version of the protocol (AS-DTMAC) aims to enhance the robustness of the background algorithm (DTMAC) against collisions by using the active signaling mechanism. Figure 1 illustrates the inclusion of this mechanism in the slot. During the signaling part of the packet, a selection process is carried out to obtain exactly one packet to be sent in the payload part of the slot.

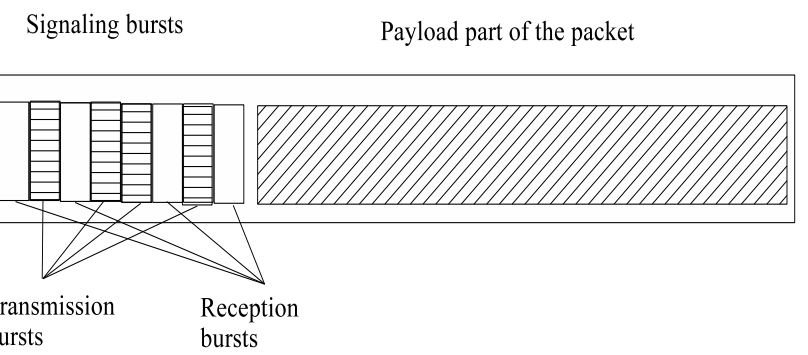

Figure 1. Slot structure of the Active Signaling mechanism

The active signaling part of the slot consists of $n$ minislots, each of which could be a transmission or a listening period. This succession is governed by a randomly generated binary key. ' 1 ' means that the vehicle with a packet to send transmits during the signaling bursts. ' 0 ' means that the vehicle with a packet to send senses the channel during this mini-slot. When a vehicle selects a listening period and senses a transmission, the competition to get the slot is over. For instance, a vehicle that draws the key '01001110' will listen during the first mini-slot and if no competing transmission is sensed during this mini-slot, it will transmit during the next mini-slot. The following two steps in the selection process will be two listening periods. The selection process continues using the same rule until the key is completely used up.

In the description above, we define the random standard access technique scheme for the active signaling. However, when a vehicle has an emergency message to transmit, the binary keys, which are initially completely random, will encompass a deterministic part represented by one bit. In this case, the vehicles that require immediate access will set the first bit to ' 1 '. Thus, these vehicles will have guaranteed priority access over the set of vehicles that are trying to get a slot using the standard scheme. These vehicles will keep the first bit set to ' 0 '.

\section{Analytical model and simulation results}

In this section, we present an analytical model proposed for AS-DTMAC and we compare the protocol performance given by this model with the simulation results. As shown in Figure 2, we have used the same simulation tools and scenarios as those used in [9]. We use MOVE and SUMO to generate vehicular traffic scenarios and to perform real vehicular mobility simulations, respectively. In our simulations we consider a digital map to build a
VANET environment close to real highway configurations taking into account lanes with different directions. This map was exported from OpenStreetMap (OSM) and adapted with the help of OpenStreetMap Editor (JOSM). The resulting roads are then populated with vehicles traveling in both directions. Each flow of vehicles is characterized by a set of parameters which consist of the starting and ending time of the flow, the initial point and the destination of the flow and the maximum number of vehicles. Table I summarizes the simulation parameters used in our experiments. As studied in [9], we have used 9 mini-slots for the signaling burst period, as it requires less computing and presents good performance.

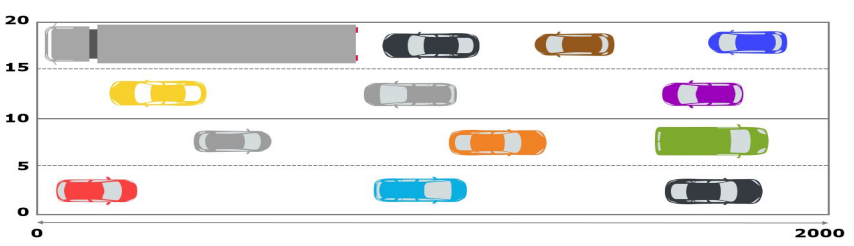

Figure 2. VANET network topology.

Table 1. Simulation parameters

\begin{tabular}{cc}
\hline Parameter & Value \\
\hline Simulation time & $100(\mathrm{~ms})$ \\
Speed & $120(\mathrm{~km} / \mathrm{h})$ \\
Speed standard deviation & $30(\mathrm{~km} / \mathrm{h})$ \\
Number of slots per frame $(\tau)$ & 100 \\
Slot duration & $0.001(\mathrm{~s})$ \\
Mini slot duration & $0.000025(\mathrm{~s})$ \\
Number of mini slots & $9[9]$ \\
Highway length & $2.5(\mathrm{~km})$ \\
The number of lanes per direction & 2 \\
The radio range $(R)$ & $310(\mathrm{~m})$ \\
\hline
\end{tabular}

In this section, we present the environment and the analytical model for the arrival process. Two cases of the arrival process can be distinguished in the AS-DTMAC protocol: in the first case, the vehicles arrive in each time slot according to a homogeneous Poisson process, while in the second case there is an arriving burst that indicates the need to transmit very urgent messages. In the first case, the vehicles try to randomly select an available time slot for their transmissions; the active signaling mechanism is used to avoid the access collision problem when two or more vehicles try to select the same slot. This is the access for the transmission of low priority messages. In contrast, the second case is when the vehicles need a low latency access to transmit their emergency warning messages. In this case, a special choice of the transmission key must be made in order to favor the urgent packets. This is the access for emergency vehicle communication.

\subsection{Homogeneous arrival}

In this section, we present an analytical model where the vehicles try to randomly select free time slots according 
to a homogeneous Poisson process in which the arrival of a transmission in each free slot is modeled as a Poisson process of rate $\lambda$. The vehicles use the FI to determine the available set of free slots and randomly select one of them. The probability that there are $k$ transmission attempts during a given time slot is:

$$
\frac{\lambda^{k}}{k !} \exp (-\lambda)
$$

We assume that we have a binary sequence of $n$ bits generated randomly by a given vehicle as its selection key. In other words, the active signaling scheme encompasses $n$ mini-slots. The goal of this study is to compute the probability that only one vehicle has been selected to transmit at the end of the selection process.

We will assume that the computation is being performed with the transmission key of a vehicle randomly generated between 0 and $2^{n}-1$. A vehicle that is still in the selection process after a transmission in a mini-slot will transmit in the next mini-slot with probability $1 / 2$ and will listen with probability $1 / 2$.

Let us suppose that we have $k$ vehicles at the beginning. Our aim is to compute the probability that $j$ vehicles are still in the selection process after the mini-slot $i$; we denote this probability by $a_{i}^{j}$. To perform this task, we use the generating function $A_{i}(x)$ of the remaining contenders after the $i^{\prime}$ th selection mini-slot. By definition we have:

$$
A_{i}(x)=\sum_{j=0}^{\infty} a_{i}^{j} x^{j} .
$$

It is easy to establish that

$$
A_{0}(x)=x^{k}
$$

And we can observe that

$$
A_{1}(x)=A_{0}\left(\frac{x}{2}+\frac{1}{2}\right)+A_{0}\left(\frac{x}{2}\right)-A_{0}\left(\frac{1}{2}\right) .
$$

We have:

$$
A_{0}\left(\frac{x}{2}+\frac{1}{2}\right)=\sum_{j=1}^{k}\left(\begin{array}{l}
k \\
j
\end{array}\right) \frac{1}{2^{k-j}} x^{k-j} \frac{1}{2^{j}}
$$

$\left(\begin{array}{c}k \\ j\end{array}\right) \frac{1}{2^{k-j}} \frac{1}{2^{j}}$ gives the probability that $k-j$ contenders transmit a burst whereas $j$ contenders are in listening mode. With this probability, the generating function must be $x^{k-j}$ which explains the transition formula ${ }^{3}$ except for the case where $j=k$ and we need a correction term to handle this. The correction term is $A_{0}\left(\frac{x}{2}\right)-A_{0}\left(\frac{1}{2}\right)$. It takes into account the fact that when the $k$ vehicles select a listening period, none of them is rejected by the selection process and we still have $k$ contenders after this step of the selection process. Similarly, by linearity, we have the recursion for $i \in$ $0, \ldots, n-1$ :

3. We have $k-j$ remaining contenders after this mini-slot

$$
A_{i+1}(x)=A_{i}\left(\frac{x}{2}+\frac{1}{2}\right)+A_{i}\left(\frac{x}{2}\right)-A_{i}\left(\frac{1}{2}\right)
$$

Thus using this recursion formula, it is easy to compute $A_{1}(x), A_{2}(x), \ldots, A_{n}(x)$, using, for instance, Maple. To show one example, we can fix $k=3$ and $n=5$. We obtain the results shown in Table 2 . After the $5^{\prime} t h$ round of mini-slot selection, the probability that there are still three contenders is $\frac{1}{1024}$, the probability that there are still two contenders is $\frac{93}{2048}$ and exactly one contender $\frac{1953}{2048}$. If the selection process stops after the $5^{\prime} t h$ round, the probability that the process is successful is :

$$
\frac{1953}{2048} \simeq 0.954
$$

and the probability of collision is:

$$
1-\frac{1953}{2048} \simeq 0.0464 \text {. }
$$

\begin{tabular}{|c|c|c|c|c|}
\hline $\mathrm{i} a_{k}^{i}$ & $a_{0}^{i}$ & $a_{1}^{i}$ & $a_{2}^{i}$ & $a_{3}^{i}$ \\
\hline$i=0$ & 0 & 0 & 0 & 1 \\
\hline$i=1$ & 0 & $\frac{3}{8}$ & $\frac{3}{8}$ & $\frac{1}{4}$ \\
\hline$i=2$ & 0 & $\frac{21}{32}$ & $\frac{9}{32}$ & $\frac{1}{16}$ \\
\hline$i=3$ & 0 & $\frac{105}{128}$ & $\frac{21}{128}$ & $\frac{1}{64}$ \\
\hline$i=4$ & 0 & $\frac{465}{512}$ & $\frac{45}{512}$ & $\frac{1}{256}$ \\
\hline$i=5$ & 0 & $\frac{1953}{2048}$ & $\frac{93}{2048}$ & $\frac{1}{1024}$ \\
\hline
\end{tabular}

Table 2. DIFFERENT PHASES OF THE SELECTION PROCESS

In the general case, the probability of collision after the end of the signaling period is

$$
\sum_{j=2}^{\infty} a_{j}^{n}=1-a_{0}^{n}-a_{1}^{n}=1-A_{n}(0)-A_{n}^{\prime}(0)
$$

since $\sum_{j=0}^{\infty} a_{j}^{n}=1$. We can note that $A_{n}(0)=a_{0}^{n}=0$. Thus if we denote by $A_{n}^{k}(x)$ the generating functions obtained with the above recursive procedure starting with $A_{0}(x)=x^{k}$, we can obtain $P_{n}(\lambda)$, the probability of collision with a signaling burst of length $n$ given that we have at least one vehicle attempting to transmit during a slot. Therefore, $P_{n}(\lambda)$ is given by the following expression:

$P_{n}(\lambda)=\frac{1}{1-\exp (-\lambda)} \sum_{k=0}^{\infty} \frac{\lambda^{k}}{k !} \exp (-\lambda)\left(1-A_{n}^{k}(0)-A_{n}^{k^{\prime}}(0)\right)$.

The analytical model presented above has been used to compute the collision probability according to the channel load. Figure 3 shows the collision probability when varying the channel load for $n=4,6,8,10$. The error bars in black are for a $95 \%$ confidence interval. We can observe that the probability of collision (around 0.02) is already low for $\lambda=$ 1 and for $n=4$. Moreover, for $n=10$ the probability of 


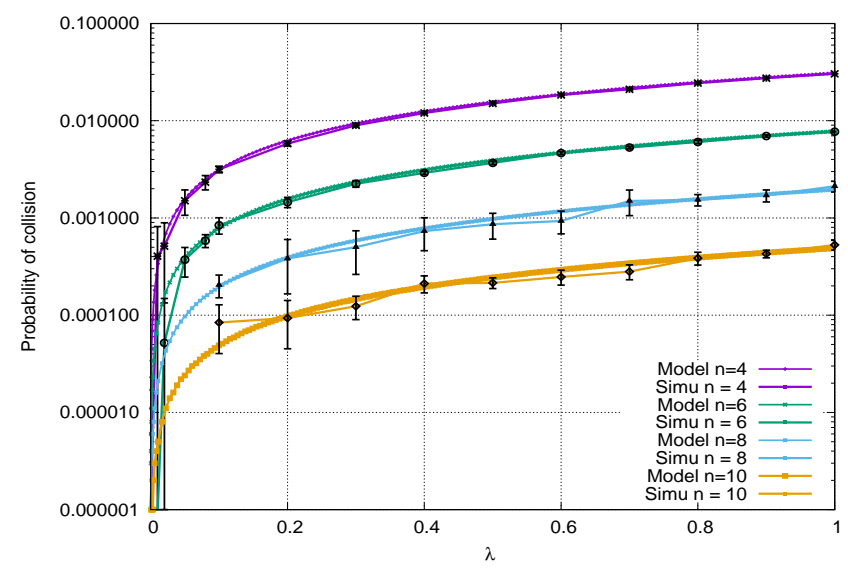

Figure 3. Collision probability versus channel load.

collision is extremely small (around 0.0005). This figure clearly shows a very good matching between the results of the analytical model and simulation.

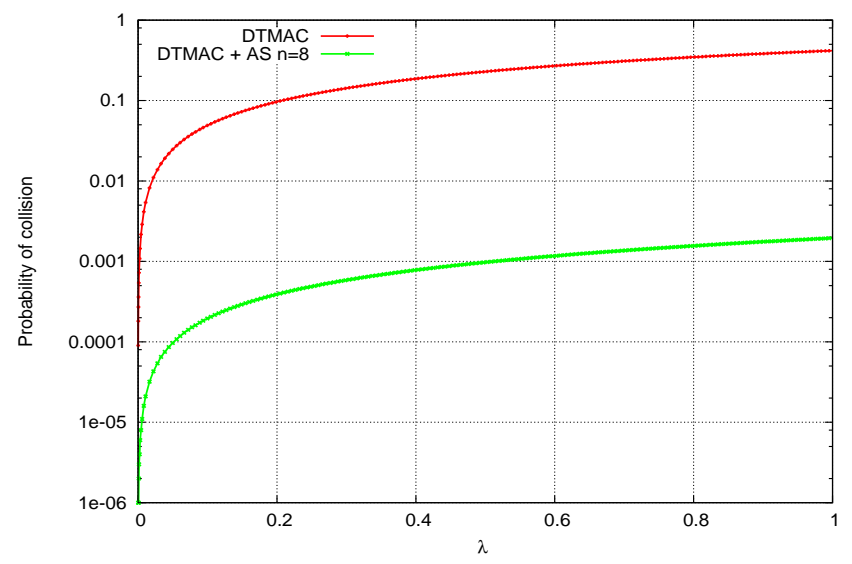

Figure 4. Collision probability vs channel load.

In Figure 4 we compare the collision probability when varying the channel load for the two protocols DTMAC and AS-DTMAC with $n=8$ mini-slots. We can observe from this figure that the gain in the probability of collision is in the order of $10^{3}$. These results clearly show the interest of the active signaling mechanism.

In this sub-section we have supposed that the detection process during the active signaling process is perfect. In the next sub-section we study the performance of the signaling process if we assume errors during the signaling. It is fundamental to verify that the signaling is robust when errors occur during this scheme and the aim of the section to show that the selection remains effective even when we assume that errors are present in the process.

\subsection{Errors in the signaling process}

3.2.1. Homogeneous arrival with errors in the signaling process. We assume again that the arrival in each slot is a
Poisson process of rate $\lambda$. Thus the probability that there are $k$ transmission attempts during this slot is still

$$
\frac{\lambda^{k}}{k !} \exp (-\lambda) \text {. }
$$

We assume that we have a binary key of $n$ bits or, in other words, the signaling scheme encompasses $n$ mini-slot intervals. We want to compute the probability that at the end of the selection process only one vehicle has been selected to transmit. We will still assume that the transmission key of a node is randomly generated between 0 and $2^{n}$, which means that after each transmission in a mini-slot a vehicle that is still in the selection process will transmit in the next minislot with probability $1 / 2$ and will listen with probability $1 / 2$.

But, here, we introduce error into the selection process. We assume that during a mini-slot selection a vehicle that is listening can miss the transmission of another vehicle with probability $\epsilon_{1}$, in other words the concurrent transmission in the mini-slot will be sensed with probability $1-\epsilon_{1}$. Conversely, during a listening period, we assume that with probability $\epsilon_{2}$ a vehicle in a listening period will sense a concurrent transmission during the mini-slot whereas there is actually no transmission. We assume that the errors occur independently.

Let us suppose that we have $k$ vehicles at the beginning. Our aim is to compute the probability that $j$ vehicles are still in the selection process after mini-slot $i$. We denote this probability by $b_{i}^{j}$. To perform this task, we use the generating function $B_{i}(x)$ of the remaining contenders after the $i^{\prime} t h$ selection mini-slot. By definition we have:

$$
B_{i}(x)=\sum_{j=0}^{\infty} b_{i}^{j} x^{j}
$$

It is easy to establish that

$$
B_{0}(x)=x^{k}
$$

and we can observe that

$$
\begin{gathered}
B_{1}(x)=B_{0}\left(\frac{1}{2} x+\frac{1}{2}\left(\left(1-\epsilon_{1}\right)+\epsilon_{1} x\right)\right)-B_{0}\left(\frac{1}{2}\left(\left(1-\epsilon_{1}\right)+\epsilon_{1} x\right)\right) \\
+B_{0}\left(\frac{1}{2}\left(\epsilon_{2}+\left(1-\epsilon_{2}\right) x\right)\right) .
\end{gathered}
$$

We have:

$$
\begin{gathered}
B_{0}\left(\frac{x}{2}+\frac{1}{2}\left(\left(1-\epsilon_{1}\right)+\epsilon_{1} x\right)\right)= \\
\sum_{j=1}^{k}\left(\begin{array}{l}
k \\
j
\end{array}\right) \frac{1}{2^{k-j}} x^{k-j} \frac{1}{2^{j}}\left(\left(1-\epsilon_{1}\right)+\epsilon_{1} x\right)^{j} .
\end{gathered}
$$

$\left(\begin{array}{l}k \\ j\end{array}\right) \frac{1}{2^{k-j}} \frac{1}{2^{j}}$ gives the probability that $k-j$ contenders transmit a burst whereas $j$ contenders are in listening mode. With this probability, the generating function must be $x^{k-j}\left(\left(1-\epsilon_{1}\right)+\epsilon_{1} x\right)^{j}$ for $j \neq k$. In fact, we have directly $k-j$ selected nodes but, among the $j$ nodes which are in listening mode, with a probability $\epsilon_{1}$ they remain selected, 
whereas, with probability $1-\epsilon_{1}$, they are rejected. This explains the transition formula except for the case where $j=k$ and we need a correction term to handle this. The correction term is:

$$
-B_{0}\left(\frac{1}{2}\left(\left(1-\epsilon_{1}\right)+\epsilon_{1} x\right)\right)+B_{0}\left(\frac{1}{2}\left(\epsilon_{2}+\left(1-\epsilon_{2}\right) x\right)\right)
$$

$-B_{0}\left(\frac{1}{2}\left(\left(1-\epsilon_{1}\right)+\epsilon_{1} x\right)\right)$ is just to remove the case where $j=k$ in the development of $\left.B_{1}\left(\frac{x}{2}+\frac{1}{2}\left(\left(1-\epsilon_{1}\right)+\epsilon_{1} x\right)\right)\right)$. The other term corresponds to the case where all the $k$ contenders are in listening mode. Since we have assumed the probability of false alarms on burst detection, for the $k$ remaining contenders, they are rejected with probability $\epsilon_{2}$ and they remain in the selection process with probability $1-\epsilon_{2}$. This explains the contribution $B_{0}\left(\frac{1}{2}\left(\left(\epsilon_{2}+\left(1-\epsilon_{2}\right) x\right)\right)\right.$ in the transition formula.

Similarly, by linearity, we have the recursion for $i \in$ $0, \ldots, n-1$ :

$$
\begin{aligned}
B_{i+1}(x)=B_{i}( & \left.\frac{1}{2} x+\frac{1}{2}\left(\left(1-\epsilon_{1}\right)+\epsilon_{1} x\right)\right)-B_{i}\left(\frac{1}{2}\left(\left(1-\epsilon_{1}\right)+\epsilon_{1} x\right)\right) \\
& +B_{i}\left(\frac{1}{2}\left(\epsilon_{2}+\left(1-\epsilon_{2}\right) x\right)\right) .
\end{aligned}
$$

Using this recursion formula, it is easy to compute $B_{1}(x)$, $B_{2}(x), \ldots, B_{n}(x)$ using, for instance, Maple.

For a Poisson arrival of rate $\lambda$ and for a given non-empty slot, we can compute the probability of collision $P r_{c o l}$ and the probability of success $P r_{s u c}$ i.e. the probability that the selection process ends with a successful transmission.

$$
\begin{gathered}
\operatorname{Pr}_{c o l}(\lambda)=\frac{1}{1-\exp (-\lambda)} \sum_{k=0}^{\infty} \frac{\lambda^{k}}{k !} \exp (-\lambda)\left(1-B_{k}(0)-B_{k}^{\prime}(0)\right) . \\
\operatorname{Pr}_{\text {suc }}(\lambda)=\frac{1}{1-\exp (-\lambda)} \sum_{k=0}^{\infty} \frac{\lambda^{k}}{k !} \exp (-\lambda) B_{k}^{\prime}(0) .
\end{gathered}
$$

It is very important to notice that the previous formulas which are valid for Poisson arrival can easily be adapted to any kind of arrival distribution given that $P(\lambda, k)$ the probability to have $k$ arrivals on given a slot can be computed. The probability of collision and the probability of success with such a general arrival law are: $\operatorname{Pr}_{\text {col }}^{g}(\lambda)$ and $\operatorname{Pr}_{\text {col }}^{\text {suc }}(\lambda)$

$$
\begin{gathered}
\operatorname{Pr}_{\text {col }}^{g}(\lambda)=\frac{1}{1-P(\lambda, 0)} \sum_{k=0}^{\infty} P(\lambda, k)\left(1-B_{k}(0)-B_{k}^{\prime}(0)\right) . \\
\operatorname{Pr}_{\text {suc }}^{g}(\lambda)=\frac{1}{1-P(\lambda, 0)} \sum_{k=0}^{\infty} P(\lambda, k) B_{k}^{\prime}(0) .
\end{gathered}
$$

We now come back to a Poisson distribution of arrivals and we study the collision probability for various loads and various numbers of mini-slots in the signaling bursts.

In Figure 5 we plot the collision probability versus the channel load for different values of the missed detection probability $\epsilon_{1}$ for $n=8$ and $\epsilon_{2}=0$. We observe a noticeable increase in the collision probability when $\epsilon_{1}$ increases, however the performance remains good even with $\epsilon_{1}=0.05$.

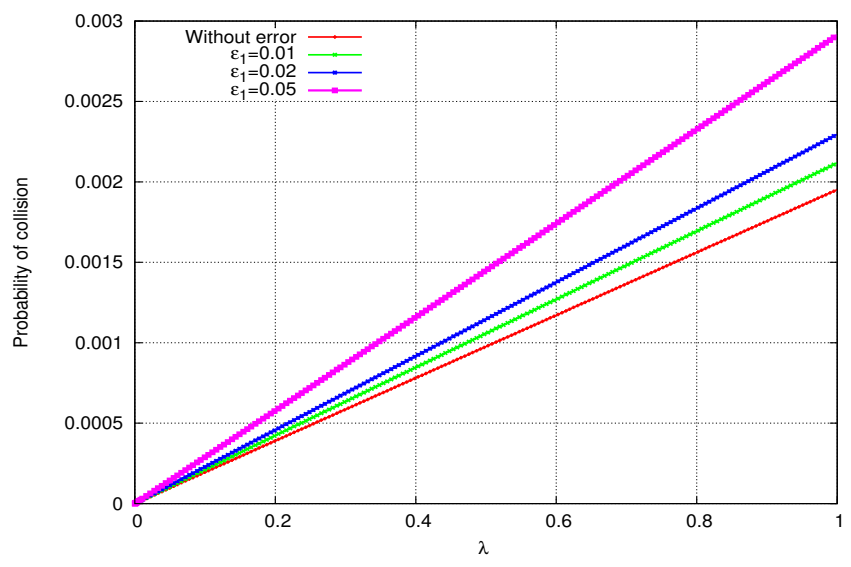

Figure 5. Collision probability with error in detecting the signaling burst $\epsilon_{1}=0.01,0.02,0.05$ (missed detection).

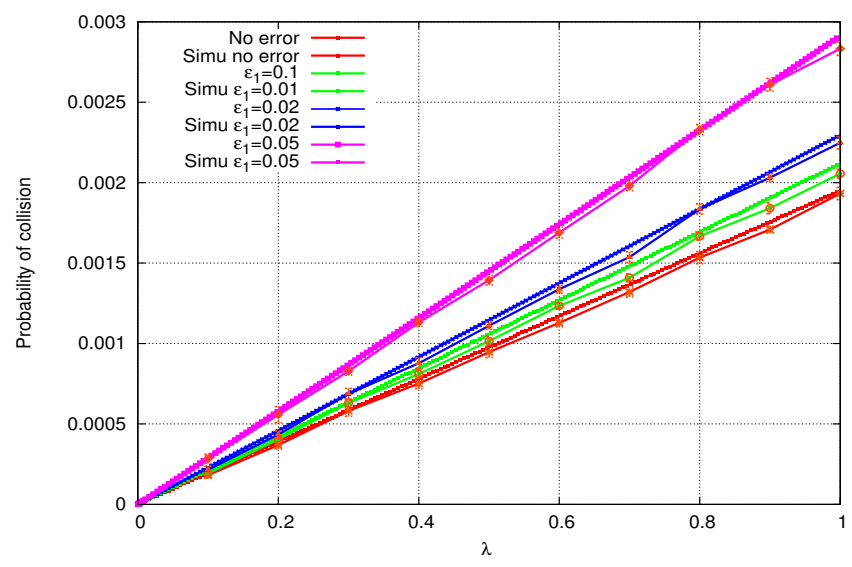

Figure 6. Collision probability with error in detecting the signaling burst $\epsilon_{1}=0.01,0.02,0.05$ (missed detection).

In Figure 6, we add the curves of the simulation results with the respective confidence interval of $95 \%$ and we keep the results of the analytical model for the comparison. For the clarity of this paper, we keep Figure 5. We can observe that there is a very good matching between the simulation results and the analytical model. In Figure 7 we plot the collision probability versus the channel load for different values of the missed detection probability $\epsilon_{2}$ and for $n=8$ and $\epsilon_{1}=0$. We observe a noticeable improvement in the collision probability when $\epsilon_{2}$ increases. This is strange since it seems that the performance improves when $\epsilon_{2}$ increases. But this can be explained by the fact that when $\epsilon_{2}>0$ there is a non-zero probability that the selection process leads to an empty slot and ends up with no transmission although there are pending packets for this slot. Figure 8 presents the comparison between the simulation results and the analytical model for the scenarios shown in Figure 7 and confirms the very good matching between the two approaches. 


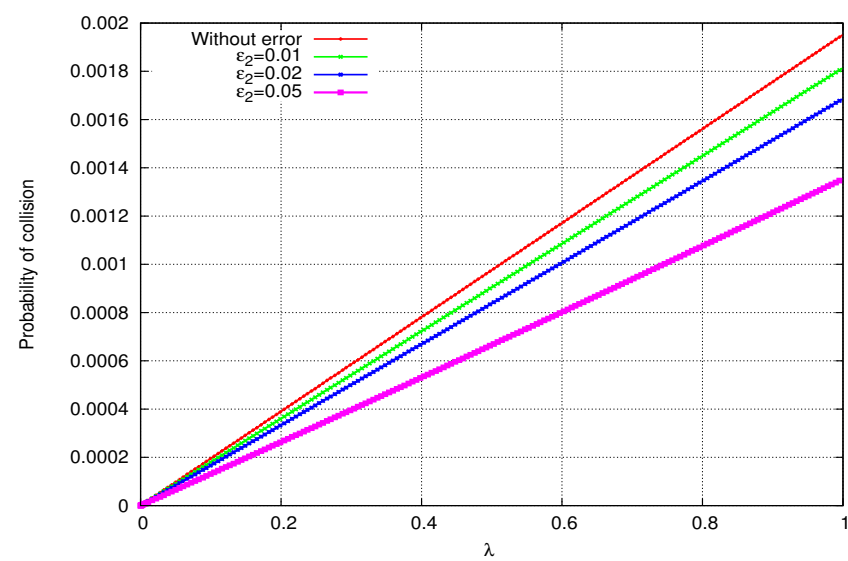

Figure 7. TBD. Collision probability with error in detecting the signaling burst $\epsilon_{2}=0.01,0.02,0.05\left(\epsilon_{1}=0.0\right)$.

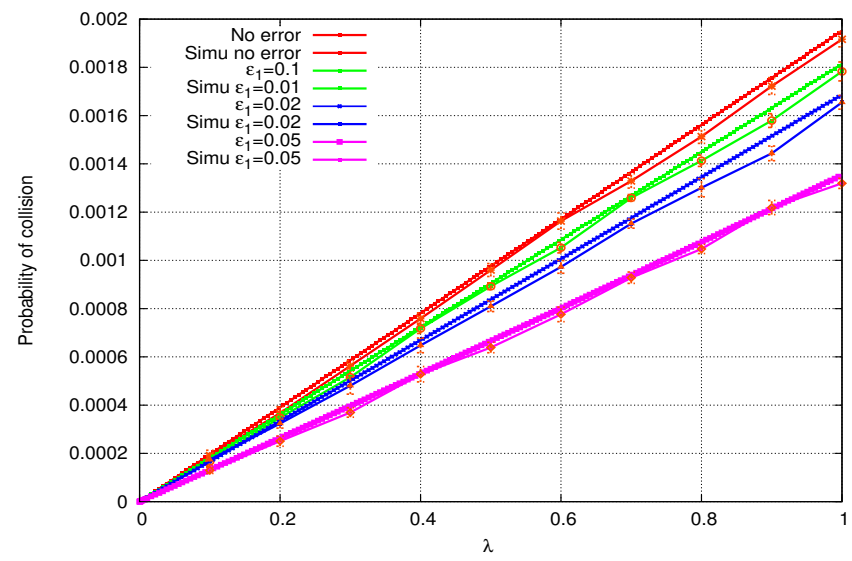

Figure 8 . TBD. Collision probability with error in detecting the signaling burst $\epsilon_{2}=0.01,0.02,0.05\left(\epsilon_{1}=0.0\right)$.

Figure 9 and Figure 10 give the probability of a successful transmission when we vary $\epsilon_{1}$ and $\epsilon_{2}$. In Figure 9, we observe that the probability of a successful transmission is nearly 1 when there is no error in the detection of the signaling sequence or when $\epsilon_{1} \leq 0.05$ and $\epsilon_{2}=0$, but, when $\epsilon_{2}=0.05$ the probability of a successful transmission falls to around 0.83 . The reason for this large decrease is not the collision rate but the occurrence of empty slots where the selection process ends with no transmission although there are pending packets for this slot. We observe that the error when phantom signaling bursts are detected is much more detrimental to the selection than the missed detection of a signaling burst. Figure 10 presents a comparison between the simulation results and the analytical model for the scenarios given in Figure 9 and confirms the very good matching between the two approaches. As an initial conclusion of this sub-section we can state that the slot selection process can cope with errors in the detection of the signaling burst.

In the next sub-section, we study how a burst of $k$ arrivals can be handled by the active signaling process. We will show that the bandwidth lost by this collision

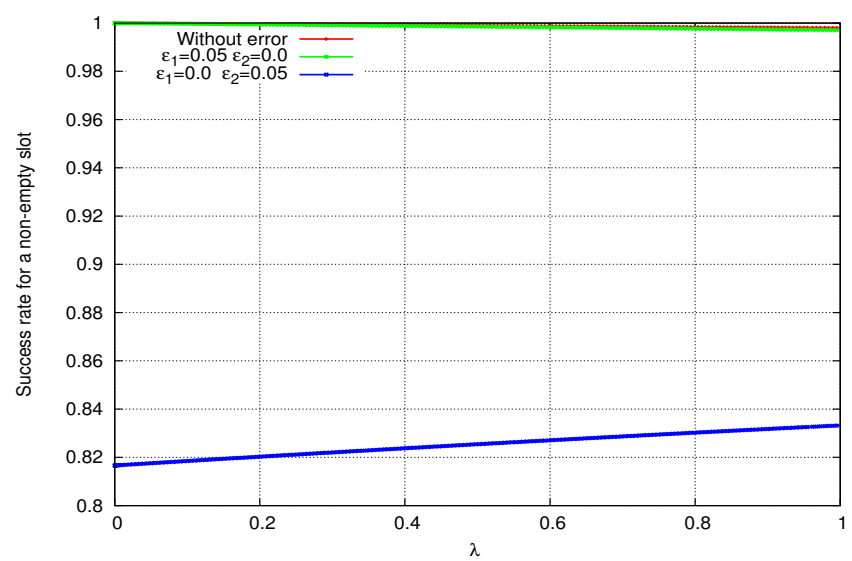

Figure 9. Probability that the selection process for a non-empty slot ends with a successful transmission for different values of $\epsilon_{1}$ and $\epsilon_{2}$.

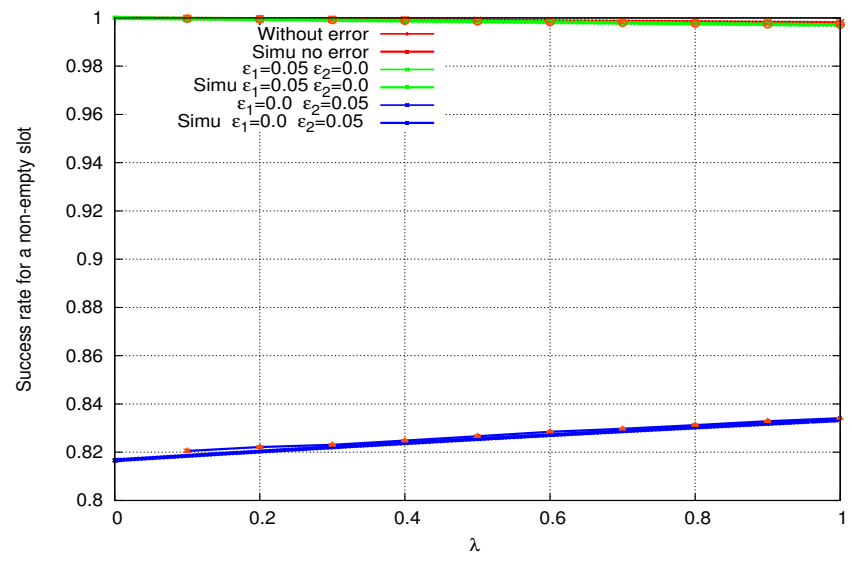

Figure 10. Probability that the selection process for a non-empty slot ends up with a successful transmission for different values of $\epsilon_{1}$ and $\epsilon_{2}$

resolution actually remains very small. Indeed, the analytical model assume that the two errors false positive and false negative follow independent process. We think that ghost transmissions are decorrelated to fading which can create detection failures of transmission mini-slots but we have not proof of that.

\subsection{Burst of traffic}

In this study, we supposed that we have a burst of $k$ arrivals of emergency messages (urgent packets) in a given slot and we assume that there are no other competitors. Such an effect can be obtained if we use a dedicated first bit in the transmission key. When a vehicle requires a priority access to the channel to transmit urgent packets, it sets the first bit in the transmission key to ' 1 '. Otherwise, it sets it to ' 0 ' to transmit default packets. By doing so, after the first signaling interval, there will be competition between $k$ urgent packets. In this section we focus on the transmission of these $k$ urgent packets. We use the simple persistent protocol to schedule these $k$ pending packets in which each 
of them successively uses the coming slot until all of the packets have been successfully transmitted. According to the computations above, the probability of collision when there are $k$ packets competing for a slot is:

$$
\epsilon_{k}=1-A_{k}(0)-A_{k}^{\prime}(0)
$$

We call $T_{k}$ the mean number of slots until the $k$ packets have been successfully transmitted. We have the following recursion

$$
T_{k}=1+\left(1-\epsilon_{k}\right) T_{k-1}+\epsilon_{k} T_{k}
$$

Thus we have the following expression:

$$
T_{k}=T_{k-1}+\frac{1}{\left(1-\epsilon_{k}\right)} .
$$

Similarly for $2 \leq j \leq k$ we can obtain:

$$
T_{j}=T_{j-1}+\frac{1}{\left(1-\epsilon_{j}\right)}
$$

and of course $T_{1}=1$. The resolution leads to

$$
T_{k}=1+\sum_{j=2}^{k} \frac{1}{\left(1-\epsilon_{j}\right)}=\sum_{j=1}^{k} \frac{1}{\left(1-\epsilon_{j}\right)} .
$$

Note that since $\epsilon_{1}=0, \frac{1}{\left(1-\epsilon_{1}\right)}=1$. Since $\epsilon_{j}$ is small, the previous equation for $T_{k}$ can re-written

$$
T_{k} \simeq k+\sum_{j=1}^{k} \epsilon_{j}
$$

We observe that the wasted bandwidth in this access procedure is directly linked to the collision probability. We have shown that this collision probability is very small. We have the inequality $T_{k}<k+k \epsilon_{k}$.

The exact distribution of $T$, the necessary number of slots until all the $k$ transmissions are successful is given by the following expression:

$$
\operatorname{Prob}(T=k)=\prod_{j=1}^{k}\left(1-\epsilon_{j}\right)
$$

$\operatorname{Prob}(T=k+m)=\prod_{j=1}^{k}\left(1-\epsilon_{j}\right) \sum_{m_{1}+\cdots+m_{k}=m} \epsilon_{1}^{m_{1}} \ldots \epsilon_{k}^{m_{k}}$

The proofs of these formulas are simple. For the first formula the probability is exactly the probability that during the $k$ successive transmissions there is no collision. Since at each transmission the number of contending nodes decreases by 1 , the result is straightforward. Note that $\epsilon_{1}=0$ is left for the symmetry of the formula.

For the second formula, the probability is exactly the probability that, to obtain $k$ successful transmissions, we encounter exactly $m$ collisions. We have to distribute these $m$ collisions into $m_{1}, \ldots, m_{k}$ collisions such as $m_{1}+\cdots+$ $m_{k}=m$ and some transmissions $j$ can be without collision, in which case $m_{j}=0$. Note that $\epsilon_{1}=0$ and that necessarily $m_{1}=0$, the terms $\left(1-\epsilon_{1}\right)$ and $\epsilon_{1}^{m_{1}}=1$ are left for the symmetry of the formula.

In Figure 11, we show the cumulative distribution function of the number of slots required for a burst of 10 urgent packets for the AS-DTMAC protocol when $n=6$ and $n=10$. We can note from Figure 11 that the AS-TDMAC protocol is very fast to transmit all the packets successfully, even for $n=6$.

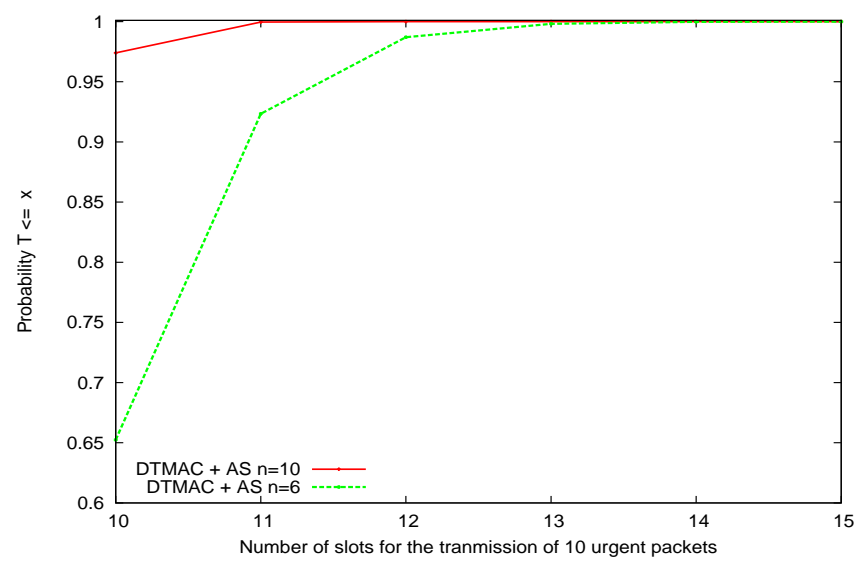

Figure 11. Cumulative distribution function of the number of slots required for the 10 urgent packets.

\subsection{Handling emergency traffic}

To handle emergency traffic, we can use the mini-slot of the signaling burst. With one mini-slot devoted to priority we can have two priority levels. The default (low priority) traffic will always be listening during the first mini-slot of the signaling burst whereas the (high priority) emergency traffic will always be transmitting during this mini-slot. In this way, high priority traffic will always have priority over a low priority packet. If we use two mini-slots devoted to priority, we can have four priority levels. These priority levels will be hierarchically independent in the sense that a packet of a higher priority will always win the competition against a traffic of a lower priority

To handle bursts of emergency traffic many options are available. The simplest technique is to use the empty slots to send these packets. The vehicles can listen to the FI field of the slots to learn the status of a slot : Free, Occupied, Collided. The emergency packets will be sent on the free or collided slots. But if a special need in terms of access delay is required, the emergency packets can be sent on occupied slots. The node whose slot hase been preempted knows that its reserved slot has been used by an emergency packet and thus it can wait for the next AS-DTMAC frame to send its packet in its reserved slot. If the emergency traffic has been sent during the previous AS-DTMAC frame, the slot will be free again for the vehicle who had an AS-DTMAC reservation for this slot. 


\subsection{Signal detection and statistics}

The aim of this part is to give an idea of how the selection process can operate at the physical layer. During a listening period, the mini-slot selection process has to choose between two hypotheses: either the signal detected contains only noise $\left(H_{0}\right)$, or there is a signal hidden in this noise $\left(H_{1}\right)$, see [25]. This process may fail; it may either fail to detect an existing signal (miss-detection) or it may detect a signal whereas there is actually only noise (false alarm).

In order to evaluate these two kinds of error, we need statistical knowledge about the distribution of the observation. Figure 12 gives an example of statistical hypothesis testing for Orthogonal Frequency Division Multiplexing (OFDM) symbol under $H_{0}$ and $H_{1}$. In the decision process, it is not uncommon that these two hypotheses are erroneously rejected. In a communication system, each detector can be characterized by the couple of $P_{f a}$ and $P_{d}{ }^{4}$ which are respectively the probability of a false alarm and the probability of detection. In practice, the probability of a false alarm is always low (less than $10^{-2}$ )[26] while the probability of detection is much greater (generally near 1) and is sensitive to the condition of the channel. According to the NeymanPearson theorem [25], the optimal detection is a Likelihood Ratio Test $(\mathrm{LRT})^{5}$ given the maximum possible $P_{d}$ for any given $P_{f a}$ [25]. This test consists of comparing the likelihood ratio to a threshold in order to make a decision. Consequently, fixing the threshold is the key to correct signal detection. For a number of applications, some parameters could be unknown while the signal is known. In this condition, we introduce a composite test approach, namely the Generalized Likelihood Ratio Test (GLRT) [25]. Generally, to obtain the best results, the detected signal is passed through a matched filter with the best possible Signal-toNoise ratio (SNR) given. We run simulations using this approach and find that the error probabilities in the bursts detection are reasonable and comparable with those used in Subsection 3.2. Therefore, we can conclude from this study that our mechanism is tolerant to $5 \%$ of false alarm errors to maintain an acceptable rate of access collision.

In Figure 13, we plot the receiver operating characteristics (ROC) curves for different SNR values: the probability of detection as a function of $P_{f a}$. These results are generated using a detection model based on GLRT. We can observe from this figure that even with a low level of signal-to-noise ratio (SNR), especially at $-13 \mathrm{db}$ when the level of $P_{f a}$ is 0.05 , the probability of detection is still high (almost $90 \%$ ).

This study will serve to configure an optimized minislot duration which ensures a sufficently low collision rate. The details of this study will be presented in a future contribution. For the active signaling application, we need a robust detection algorithm which ensures that the transmissions of the mini-slots are correctly detected. Actually, most detection implementations are based on the energy detection

4. The probability of miss-detection is $1-P_{d}$

5. We had many fruitful discussions with our colleague Cedric Adjih on the subject.

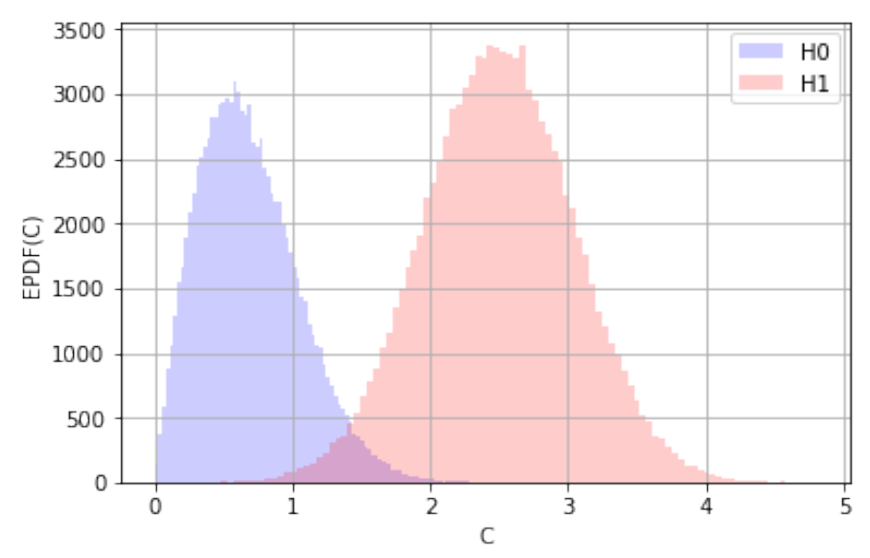

Figure 12. Example of hypothesis testing for OFDM symbol detection: Empirical Probability Distribution Function (EPDF) histogram under $H_{0}$ and $H_{1} . \mathrm{C}$ is the correlation variable at the matched filter output.

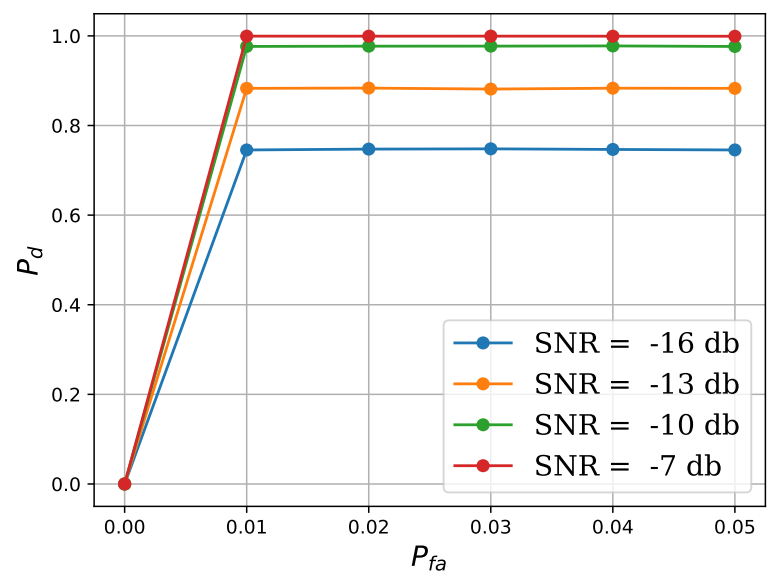

Figure 13. ROC curves for different SNR values

(ED) model due to its low computational cost. However, unlike ED, GLRT can detect below the threshold of the receiver sensitivity, which is very useful for our application. Figure 14, shows a comparision between the probabilities of detection of ED and GLRT. We observe that the detection capability of GLRT under noisy conditions greatly exceeds that of ED.

\section{Conclusion}

We present an analytical model for studying the performance of the AS-DTMAC protocol. We use recursive equation on generating functions to obtain the probability of collision. Two cases of the arrival process in AS-DTMAC have been investigated and modeled. In the first case, the packets from the vehicles arrive in each time slot according to a homogeneous Poisson process, whereas in the second case there is a burst of arrivals for very urgent messages. To make our analytical model as complete as possible, we 


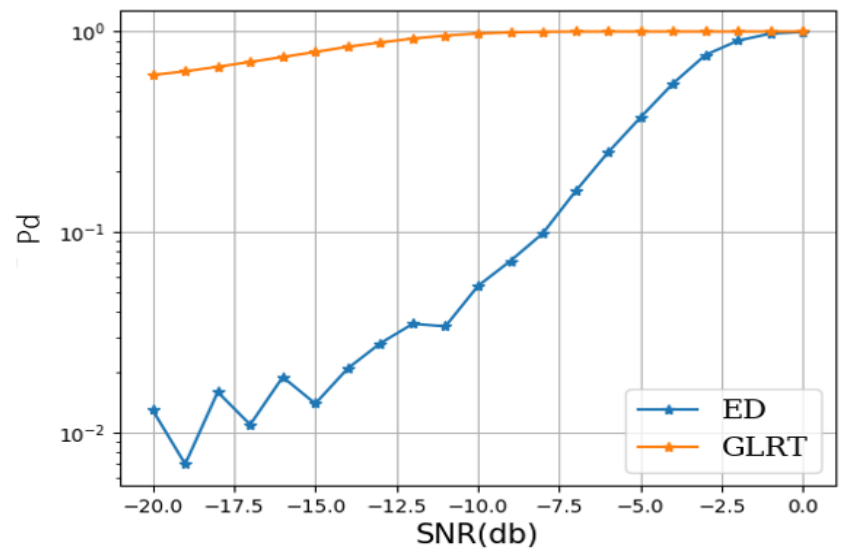

Figure 14. Performance comparison of GLRT against ED

introduce error into the model during the selection process. We define two kinds of error that can occur in the detection process: vehicles can either miss a transmission or sense a false transmission. With the first kind of error, the performance remains very good, even with up to $5 \%$ of error, whereas with the second kind of error, the selection may end up with no transmission on the payload slot, which is much more detrimental to the whole performance.

The simulation results confirm the validity of the analytical model and show that AS-DTMAC very significantly outperforms the DTMAC protocol in terms of collision probability. The transmission of urgent packets is also very efficient in AS-DTMAC. In future work, we plan to precisely evaluate the probability of the error in the detection of bursts in order to optimize the active signaling process. Furthermore, we plan to investigate the idea of no longer considering that the CSMA-based MAC protocols and TDMAbased MAC protocols are competitors. By combining the two techniques, the drawbacks of contention based-access in the former and resource reservation in the latter can be overcome. Such an application could be the future Wi-Fi standard for vehicles (IEEE 802.11bd [24]), which requires ensuring a backward compatibility mode (to make communication possible between vehicles using IEEE 802.11p and vehicles using the new communication technologies (IEEE 802.11bd). Proposing this kind of protocol will be beneficial: the CSMA/CA will keep communication with older nodes (IEEE $802.11 \mathrm{p}$ ) possible, while AS-DTMAC will enable low latency access.

\section{References}

[1] N. Lu, N. Cheng, N. Zhang, X. Shen and J. W. Mark, Connected vehicles: Solutions and challenges, IEEE internet of things journal, vol. 1, no. 4, pp. 289-299. 2014.

[2] C.D. Wang, and J.P. Thompson, Apparatus and method for motion detection and tracking of objects in a region for collsion avoidance utilizing a real-time adpative probabilistic neural network, U.S patent no. 5.613.039, 1997.

[3] K. Pi. Chen and P. A. Hsiung, Vehicle Collision Prediction under Reduced Visibility Conditions, Sensors journal, Vol. 18, no. 9, Sep 2018.
[4] S. A. Mohammad, A. Rasheed and A. Qayyum, VANET architectures and protocol stacks: a survey, In International Workshop on Communication Technologies for Vehicles, Berlin, Heidelberg, pp. 95-105, Mar. 2011.

[5] Y. Maalej, S. Sorour, A. Abdel-Rahim and M. Guizani, Vanets meet autonomous vehicles: Multimodal surrounding recognition using manifold alignment, IEEE Access, vol. 6, pp. 29026-29040, May. 2018.

[6] H. Menouar, F. Filali and M. Lenardi, A survey and qualitative analysis of MAC protocols for vehicular ad hoc networks, IEEE wireless communications, vol. 13, no. 5, pp. 30-35, 2006.

[7] W. Zhuang, H. A. Omar and L. Lio, Vemac: A novel multichannel mac protocol for vehicular ad hoc networks, in IEEE Conference on Computer Communications Workshops (INFOCOM WKSHPS), Shanghai, China, pp. 413-418, Aug. 2011.

[8] M. Hadded, A. Laouiti, P. Muhlethaler and L. A. Saidane, An infrastructure-free slot assignment algorithm for reliable broadcast of periodic messages in vehicular ad hoc networks, in Vehicular Technology Conference VTC-Fall, Montreal, Canada, Sep. 2016.

[9] F. Boukhalfa, M. Hadded, P. Muhlethaler and O. Shagdar, An Active Signaling Mechanism to Reduce Access Collisions in a Distributed TDMA Based MAC Protocol for Vehicular Networks, In International Conference on Advanced Information Networking and Applications, Matsue, Japan, vol. 926, pp. 286-300, Mar. 2019.

[10] D. N. M. Dang, H. N. Dang, V. Nguyen, Z. Htike and C. S Hong, HER-MAC: A hybrid efficient and reliable MAC for vehicular ad hoc networks, IEEE 28th International Conference on Advanced Information Networking and Applications, pp. 186-193, May. 2014.

[11] M. Hadded, P. Muhlethaler, A. Laouiti, R. Zagrouba, and L. A. Saidane, TDMA-based MAC protocols for vehicular ad hoc networks a survey, qualitative analysis and open research issues, IEEE Communications Surveys Tutorials, vol. 17, no. 4, pp. 2461-2492, Jun. 2015.

[12] F. Ye, R. Yim, J. Zhang, and S. Roy, Congestion control to achieve optimal broadcast efciency in vanets, in IEEE International Conference on Communications (ICC), Cape Town, South Africa pp. 1-5, May 2010.

[13] 802.11p-2010, IEEE standard for information technology - Telecommunications and information exchange between systems - local and metropolitan area networks - specific requirements part 11 : Wireless LAN medium access control (MAC) and physical layer (PHY) and physical layer (PHY) specifications amendment 6 : Wireless access in vehicular environments Std., 2010.

[14] R. Uzcategui and G. Acosta-Marum, Wave: A tutorial, IEEE Communications Magazine, vol. 47, no. 5, pp. 126-133, May 2009.

[15] Q. Chen, D. Jiang and L. Delgrossi, IEEE 1609.4 DSRC multi-channel operations and its implications on vehicle safety communications, IEEE vehicular networking conference (VNC), pp. 1-8, Oct. 2009.

[16] V. Nguyen, C. Pham, T. Z. Oo, N. H. Tran, E. N. Huh and C. S. Hong, MAC protocols with dynamic interval schemes for VANETs, Vehicular Communications, vol. 15, pp. 40-62, 2019.

[17] F. Borgonovo, A. Capone, M. Cesana, and L. Fratta, Adhoc mac: new mac architecture for ad hoc networks providing efficient and reliable point-to-point and broadcast services, Wireless Networks, vol. 10, no. 4, pp. 359-366, 2004.

[18] F. Borgonovo, A. Capone, M. Cesana and L. Fratta, Rr-aloha, a reliable r-aloha broadcast channel for ad-hoc intervehicle communication networks, in IEEE IFIP Annual Mediterranean Ad Hoc Networking Workshop (Med-Hoc-Net), Baia Chia, Italy, 2002.

[19] R. Zou, Z. Liu, L. Zhang and M. Kamil, A near collision free reservation based MAC protocol for VANETs, IEEE Wireless Communications and Networking Conference (WCNC), pp. 1538-1543, Apr. 2014.

[20] W. D. YANG, L. I. Pan and H. S. ZHU, Adaptive TDMA slot assignment protocol for vehicular ad-hoc networks, The Journal of China Universities of Posts and Telecommunications, vol. 20, no. 1, pp. 11-25, 2013 
[21] W. Yang, W. Liu, P. Li and L. Sun, TDMA-based control channel access for IEEE 802.11 p in VANETs, International Journal of Distributed Sensor Networks, vol. 10, no. 8, pp. 579-791, 2014.

[22] S. Babu, M. Patra and C. S. R. Murthy, An efficient TDMA-based variable interval multichannel MAC protocol for vehicular networks, Wireless Networks, vol. 22, no. 44, pp. 1365-1380, 2016.

[23] F. Boukhalfa, M. Hadded, P. Muhlethaler and O. Shagdar, An Analytical Model for Performance Analysis of An active Signalingbased MAC Protocol for Vehicular Networks, in Vehicular Technology Conference VTC-FALL, Honolulu, Hawaii, USA, Sept. 2019.
[24] G. Naik,B. Choudhury and J. M Park, IEEE 802.11 bd \& 5G NR V2X: Evolution of Radio Access Technologies for V2X Communications, IEEE Access journal, vol. 7, pp. 70169-70184, May. 2019.

[25] H. V. Poor, An introduction to signal detection and estimation, Springer Science \& Business Media, 2013.

[26] G. S. M. Motorola, 10-Estimation de probabilités faibles en détection, 1998. 